\title{
ASSEMBLY AND DYNAMICS TESTS OF NEWLY DESIGNED FLEXIBLE RAILWAY WHEELSET
}

\author{
Ilkham Galiev $^{\mathrm{a}}$, Alexander Shiler ${ }^{\mathrm{a}}$, Valeriy Shiler ${ }^{\mathrm{a}}$ \\ ${ }^{a}$ Omsk State Transport University, Karl Marx Ave. 35, Omsk 644046, Russia
}

\begin{abstract}
Technical development of rail transport systems is directed on increase of traffic safety level and decrease in expenses of rolling stock functioning. A key problem of all railroads is a decrease in dynamic forces in system "wheel-rail". The new design of wheelset with independent rotation of all points of contact with a rail - "A flexible wheel" can become the breakthrough decision. Model and computer simulation showed a number of advantages of the Flexible wheel over the standard construction. The article describes design researches and technological features of new wheelset production.
\end{abstract}

Keywords: Flexible wheelset, energyefficient railway transport, highspeed railway transport.
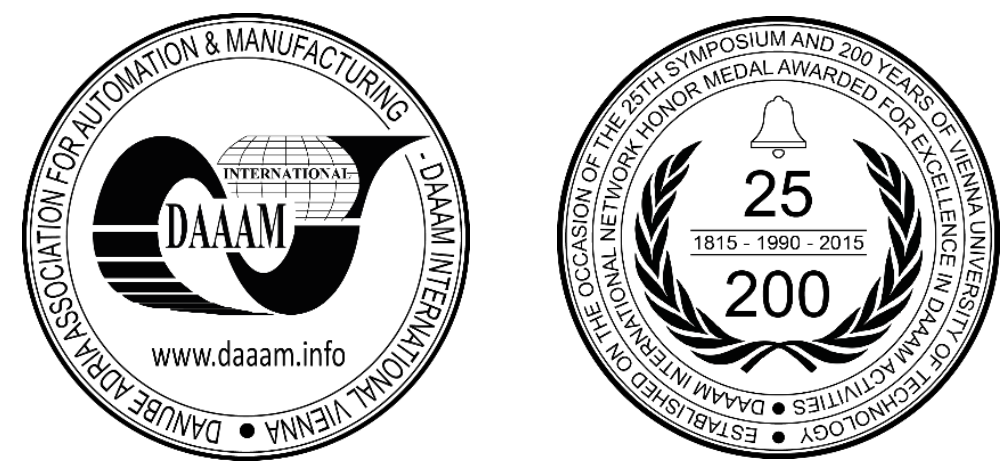

This Publication has to be referred as: Galiev, I[lkham]; Shiler, A[lexander] \& Shiler, V[aleriy] (2016). Assembly and dynamics tests of newly designed flexible railway wheelset, Proceedings of the 26th DAAAM International Symposium, pp.1171-1178, B. Katalinic (Ed.), Published by DAAAM International, ISBN 978-3-902734-07-5, ISSN 1726-9679, Vienna, Austria

DOI: 10.2507/26th.daaam.proceedings. 165 


\section{Introduction}

Increasing carrying capacity of the railways is carried out mainly due to the growth of load in the «wheel - rail» system. The work on the limit of the durability using causes increased defectiveness and intensive wear of wheels and rails [1]. The current situation explained by the fact that with increasing of axle load conditions for reducing tension weren't created in the «wheelset - rail track» system. One of the ways to solve this problem is a new wheelset design with independent rotation of the supporting and guiding elements of the wheelset wheel block. The proposed design has the following advantages over standard wheelset [2]: parasite sliding lack of all wheelset wheels on rails and tortuous motion path of the wheelset, reduced resistance to the moving along of rolling stock and, as a consequence, reduced impact on the path.

\section{Wheelset axle}

The standard axle "Py1-950" used as wheelset axle of the new design. At the present time this axle has shown high reliability during long lifetime, the technology of its manufacturing and repair is elaborated. The use of seriesproduced wheelset axle, checked in operation, positively influences on reliability of the new wheelset design as a whole.

\section{Wheel block}

Wheel in new wheelset design is made in the form of a wheel block, which consists of two elements: supporting and guiding wheels. Supporting wheel consists of the wheel centre and flexible tire, which is mounted on the wheel centre by means of an elastic pad (rubber). Running surface of flexible tire has a cylindrical shape without flange. The supporting wheel is mounted on the wheelset axle by means of two wheel bearings and adapted to transmit axle loads on the rail. Such installation of the supporting wheel on the axles ensures its independent rotation relative to all elements of the new wheelset design. The steering wheel has the form of the standard wheel flange on the perimeter and pressed onto wheelset axle on indicator diagram. The independent rotation possibility of all wheelset wheels eliminates the need for traversed path alignment of the supporting wheels and allowed to replace the conical running surface on cylindrical.

\subsection{Materials of the wheel block parts}

Elements of supporting and guiding wheels are made from the same steel grade as the standard wheelset wheel. As the material of elastic shim between the wheel centre and a flexible tire is used rubber, which applies in a silent block of the journal box locking bolt of the electric locomotive wheelset.

\section{Computer model}

Prototyping and computer models were created for three variants of the supporting wheel designs, which are discussed below. For all execution variants of the proposed wheelset design the basic geometric sizes and strength parameters of materials correspond to the parameters of the standard wheelset and GOST requirements [2,3].

Epures of the volume-stressed state, obtained by finite element method, were used to optimize the form of new wheelset construction elements. The estimated model is a half element under investigation, obtained by cutting off the vertical plane, passing through the axle and the centre of wheel contact patch with rail. In the calculation it is assumed that the vertical longitudinal symmetry plane of the wheel coincides with the vertical plane of the rail symmetry. Value of the contact patch square with the rail assumed constant in order to reduce the dimension of the task. The rail impact is not taken into account in the calculations.

The boundary conditions, imposed on the model, limit its free displacement as a whole and replace details that do not participate in the calculation, by interaction forces with them. Limitations of the «Symmetry» type, that prohibit the normal displacement to the face, superimposed on face, obtained via original model section by the vertical plane, since only half of the wheel is considered in the calculations. The opening for the installation of the outer bearing ring have restrictions, forbidding radial and axial displacements of the cylindrical face.

In place of the «wheel - rail» contact patch is applied radial force $112.8 \mathrm{kN}$. Since the calculation is performed for half a wheel, the force should be divided into two parts equal to $56.4 \mathrm{kN}$. In addition, the cross wheel slip force on the rail head acting to contact patch is defined by the following formula:

$$
F_{\mathrm{oc}}^{\max }=F_{\text {тр }}=\mu \cdot N
$$

Nomenclature
$\mu \quad$ Coefficient of sliding friction of steel on steel is 0.2
$\mathrm{~N} \quad$ Reaction force from the rolling stock weight attributable to one wheel is $112.8 \mathrm{kN}$


After substituting the values into the formula 1, the value of the axial force for the wheel half is obtained and equals $22.56 \mathrm{kN}$.

Application of load carried out in stages in the middle of calculation. At first, the radial load increases from 0 to $112.8 \mathrm{kN}$, and then thereto the axle load is gradually added from 0 to $22.56 \mathrm{kN}$. Such combination of the wheel load corresponds to the most adverse operating conditions that may occur during operation. Unfortunately, the solving program doesn't allow the application of force to different effort increasing charts in time to the same face (contact patch square). Therefore, the axial force applied to other facets of small square, highlighted on the running surface of flexible tire and adjacent to the conventional contact patch square with the rail.

Because the computer model consists of three details (wheel centre, flexible tire and elastic pad), it is necessary to impose contact conditions «Connected» on the mating facets of the individual parts, which corresponds to metal and rubber gluing during vulcanization. In solving the problem «Nonlinear» solver was used. This choice is due to fact that there is significant deformation of the rubber pad, since its elastic modulus is several orders less than that of the steel details and also the load is a time function.

The convergence of the computing process was provided with the following program solver settings:

- More sustainable and resource-intensive direct solver Direct Sparse was used,

- The use of the algorithm for nonlinear geometry which takes into account large displacements and deformations in the decision,

- Simplified incompatible communication parameters,

- The Newton-Raphson method of iteration,

- The convergence tolerance is 0.001 ,

- Eliminating coefficient of singular point of 0 (zero).

Finite element mesh, constructed on the basis of the curvature, and finite element mesh with 16 Jacobian points, allowing the hems of the finite elements have a parabolic shape, were used in the calculations. In addition, the grid control element is used in place of the supposed stress concentration of the wheel centre metal and flexible tire, and also the rubber pad, which is subjected to the greatest deformation.

General view and finite element mesh with marked boundary conditions for the wheel of the first variant are shown in Fig. 1 and Fig 2. The volume-stressed state (Fig. 3) and the resulting displacements (Fig. 4) were obtained by calculation. Deformation of the support wheel elements are presented the true scale 1:1.

At the epure of the volume-stressed state (Fig. 3), it can be seen that as a result of application of the radial and axle eccentric load greatest stresses occur in the transition region of the rim inner ring to the thinner middle part of the wheel centre. Stresses reaches values of $268 \mathrm{MPa}$, which is less than the allowable value of 291.2 MPa. In the rubber pad the stresses reaches 2.6 MPa, which is less than the tensile strength for this rubber brand (17 MPa). The resulting displacement (Fig. 4) shows that the lower part of the flexible tire, to which shear force is applied, is exposed to the greatest displacement. Deformation of the rubber pad is shown the true scale 1:1 and the axial displacement of the flexible tire bottom point is $6 \mathrm{~mm}$. It is necessary to take into account in wheel block configuration and selection of gap value between the supporting and guiding wheels, which should be at least $7 \mathrm{~mm}$ to avoid their contacting during displacement.

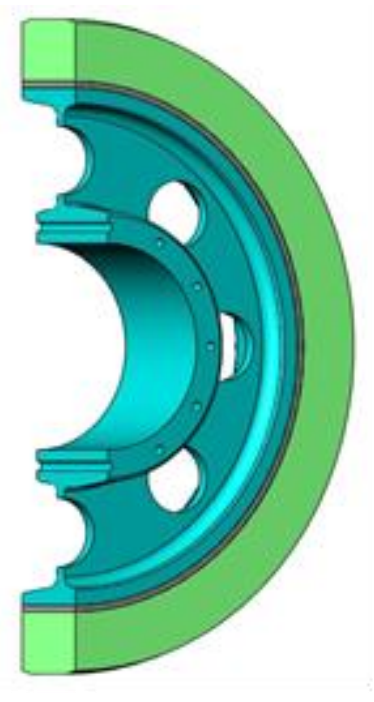

Figure 1 - Computer model of the support wheel (variant 1)

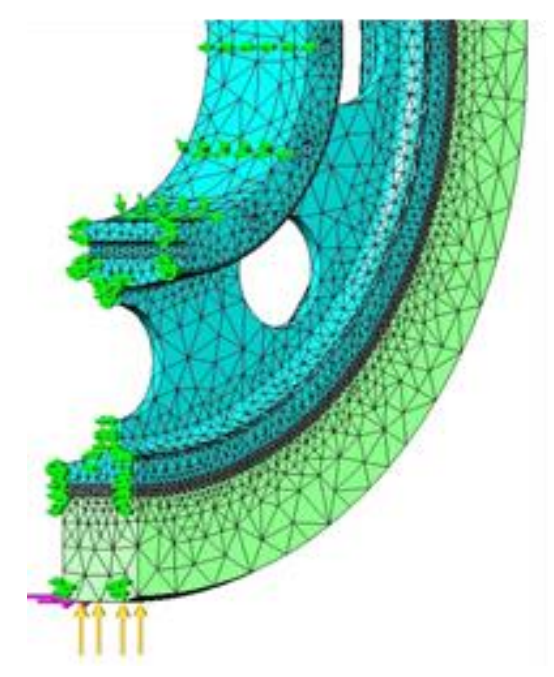

Figure 2 - Finite element mesh and boundary conditions of the support wheel model (variant 1) 


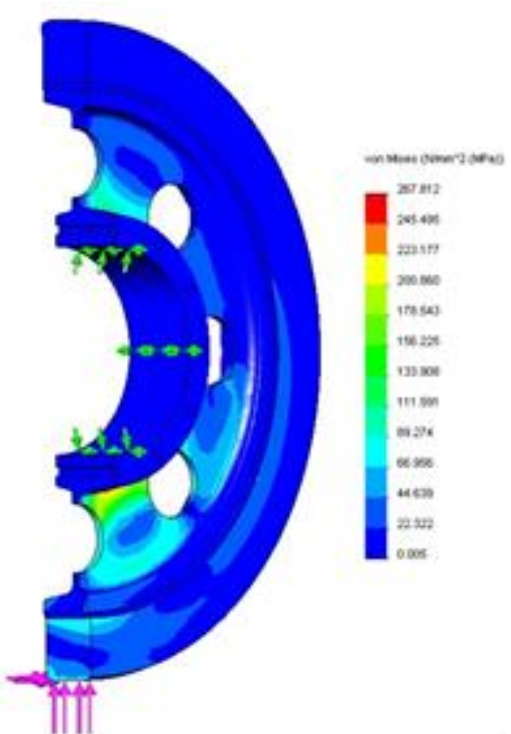

Figure 3 - Volume-stressed state of the supporting wheel (variant 1)

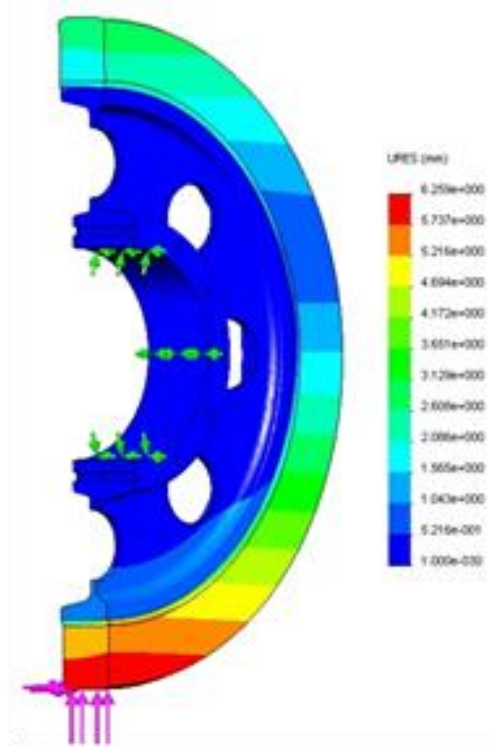

Figure 4 - Resulting displacements of the supporting wheel (variant 1))

Based on the above, supporting wheel with a rubber pad of rectangular section has the following advantages:

- Easy and manufacturable structural elements form of supporting wheel,

- Ease of assembling,

- Significant deformation energy dissipation that reduces the level of stresses in the wheel centre and allow to reduce unsprung weight value, supplied to contact patch with the rail.

Essential disadvantages of the first construction variant of the supporting wheel are:

- Low stiffness rubber pad in the axial direction that leads to substantial its deformation and, accordingly, excessive heat,

- Accelerated aging of the rubber pad,

- Possibility of the flexible tire coming off to the wheel centre in case of destruction of the rubber pad.

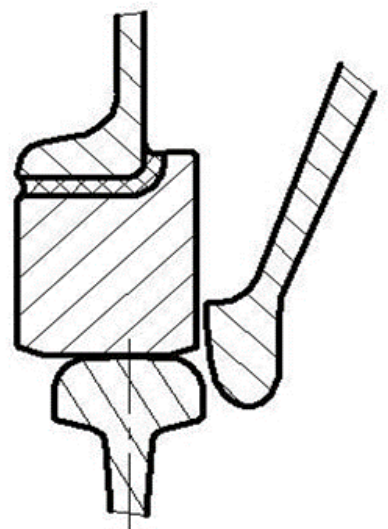

Figure 5 - Connection of the wheel centre and supporting wheel flexible tire according to tongue and groove the scheme (variant 2)

Other two variants of the mating surfaces form of the wheel centre and flexible tire, which would exclude their separation in the rubber pad destruction, were considered to enhance traffic safety of a new wheelset design.

The second variant of the supporting wheel design is based on the use of the tongue and groove system Fig. 5, which eliminates the possibility of a flexible tire creeping from the wheel centre in case of elastic pad destruction. This design has several significant drawbacks. They include the availability of a complex load of the compression and shear in bending elastic rubber pad that is the reason of uneven stiffness formation of its edges. It inevitably leads to a flexible tire skewed relative to the wheel centre, as well as to an increase in deformation and stress concentration in the metal in the rounding area of the tongue and groove system. 
The third variant is more rational: to impart a convex shape to running surface of flexible tire that contacts with the rubber pad and a concave shape to wheel centre surface. Then the rubber pad also will take the appropriate form (Fig. 4), but without sharp bends, and will constantly hold the flexible tire in a predetermined position relative to the longitudinal wheel centre plane.

In the case of the rubber pad destruction the guaranteed axle overlap between mating surfaces edges of the flexible tire and the wheel centre prevents the flexible tire coming off from the wheel centre. The axle overlap between the mating surfaces edges is provided by values overlap of their radii and flexible tire preheating with its installation on the wheel centre. Then the free space between their mating surfaces is filled with a rubber mixture and vulcanized.

Edges radius of the inner surface of flexible tire is determined from the formula:

$$
R^{\prime}=\frac{L+\Delta L}{2 \pi}
$$

Flexible tire elongation when heated is determined by the equation:

$$
\Delta L=K \cdot L \cdot \Delta \mathrm{T}
$$

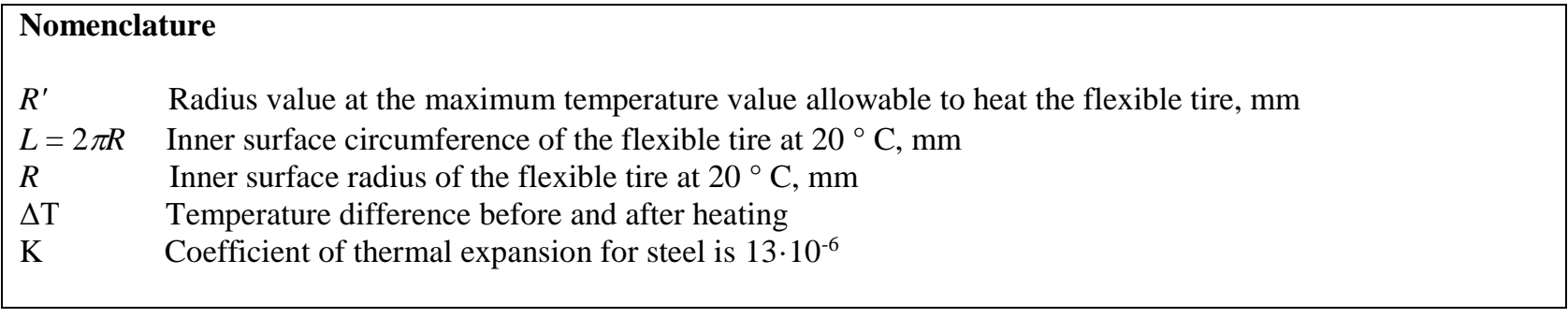

The minimum covering of the flexible tire inner surface and wheel centre is $5 \mathrm{~mm}$. The inner concave surface curvature of the flexible tire and the convex outer surface of the wheel centre was determined using the formula (2) and it is $75 \mathrm{~mm}$.

General view of the computer model and finite element mesh with marked boundary conditions for the supporting wheel execution in the third variant are shown in Fig. 6 and Fig. 7. Volume-stressed state (Fig. 8) and the resulting displacements (Fig. 9) were obtained as a result of calculations. Strain values are presented the true scale 1:1.

Under the action of the radial and eccentrically applied axle loads the largest stresses occur in the transition region of the rim inner ring to the thinner wheel centre middle part [4]. Stresses reach 253.5 MPa, which is less than the allowable value 291.2 $\mathrm{MPa}$. The stresses in the rubber pad increased to $2.5 \mathrm{MPa}$, which is much less than the tensile strength for this rubber brand (17 MPa) [5].

The stiffness of the flexible tire increased in the axial direction, since the rubber pad become to run not only on shift, but also a compression under the action of shear forces. It has led to inconsiderable increase of stress in the wheel centre, so it was necessary to increase the rounding radius in the transition areas of the rim inner ring of the wheel centre to the its middle part and reduce the technological holes in the middle part. Wheel weight has remained unchanged relative to the first variant.

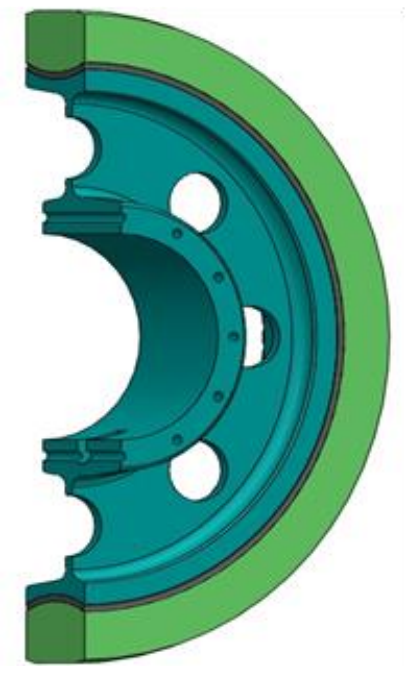

Figure 6 - Computer model of the supporting wheel (variant 3)

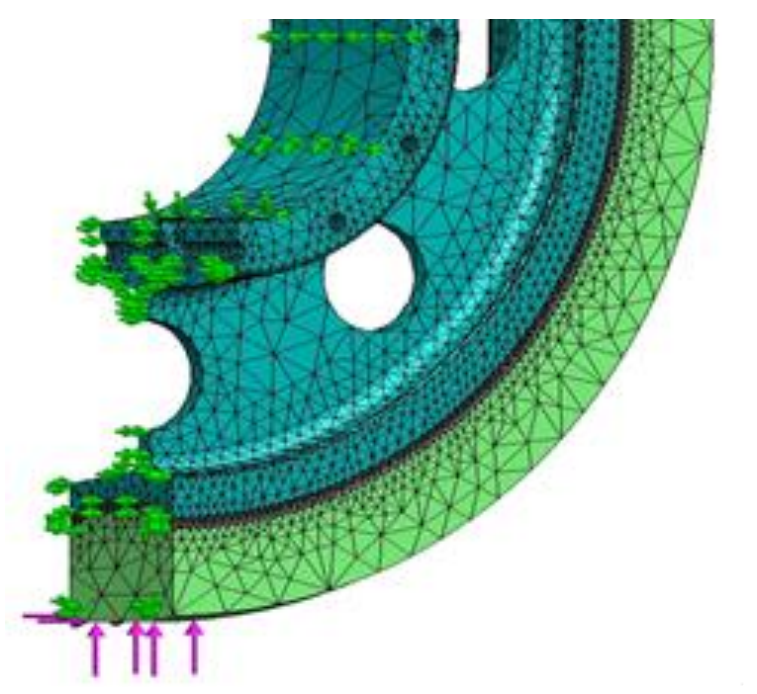

Figure 7 - Finite element mesh and boundary conditions of the supporting wheel model (variant 3) 
According to the resulting displacement (Fig. 9), the bottom part of the flexible tire, to which is acted shear force is subject to the largest displacement. The axial displacement of the flexible tire bottom point has reduced compared with the first variant to $4.4 \mathrm{~mm}$. Therefore, the gap between the resistant and supporting wheels can be reduced from 7 to 5 $\mathrm{mm}$. The axial stiffness level of the flexible tire managed to significantly increase without magnification the stress level in it and supporting wheel mass due to curved shape allotment to mating surfaces of the flexible tire and the wheel centre, as well as to increase reliability and traffic safety.

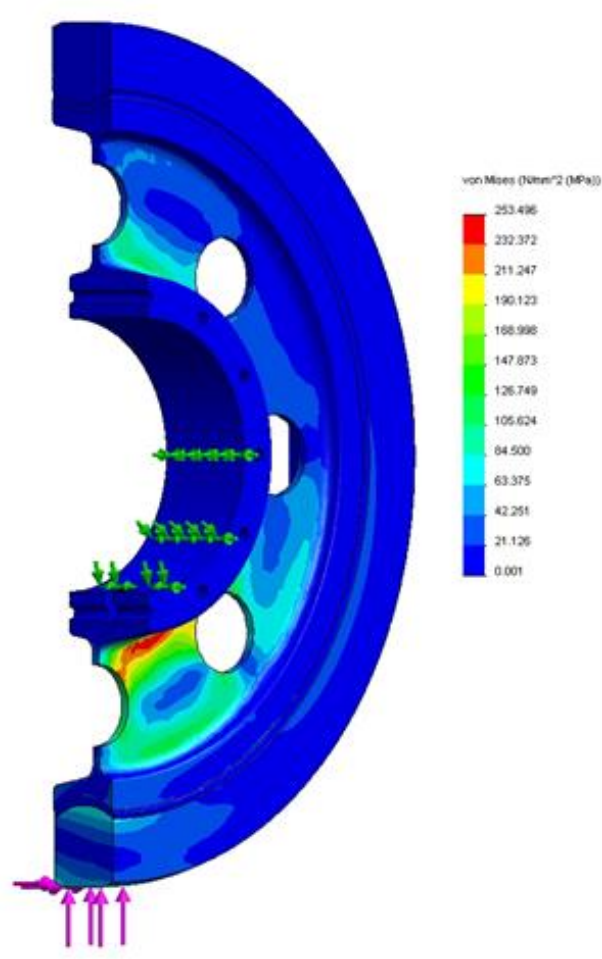

Figure 8 - Volume-stressed state of the supporting wheel (variant 3)

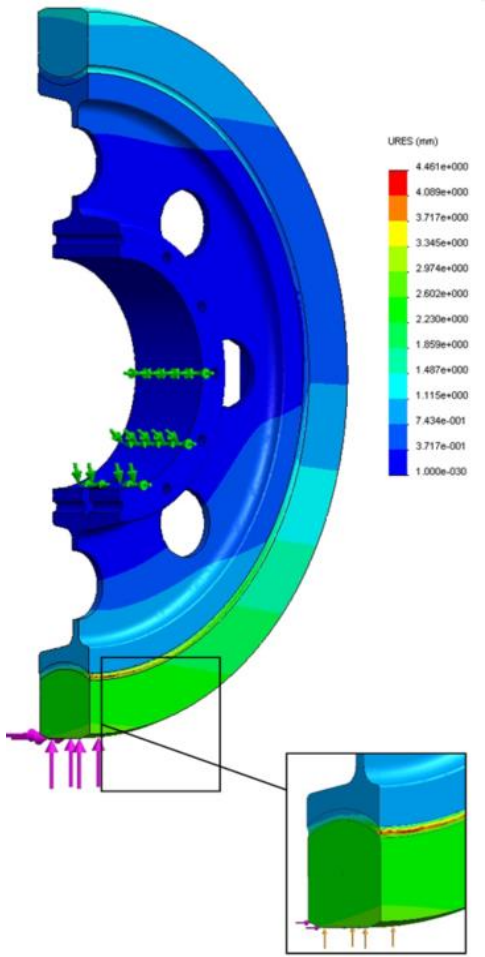

Figure 9 - Resulting displacements of the supporting wheel (variant 3)

To reduce the wheel block weight and wheelset as a whole, it was decided to develop a wheel block with a diameter of the flexible tire surface equals $780 \mathrm{~mm}$. This surface diameter corresponds with international experience of high-speed rolling stock exploitation.

Overall view and finite element mesh with marked boundary conditions for wheel, performed in the third variant with a diameter of the flexible tire surface equals 780 mm, is shown in Fig. 10 and Fig. 11.

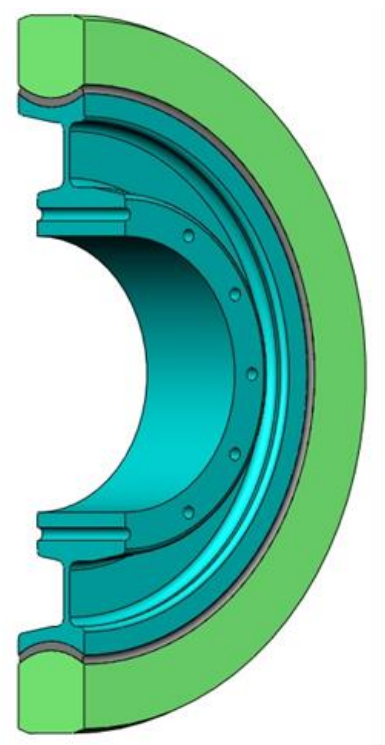

Figure 10 - The computer model of the supporting wheel (variant 3) with a diameter $780 \mathrm{~mm}$

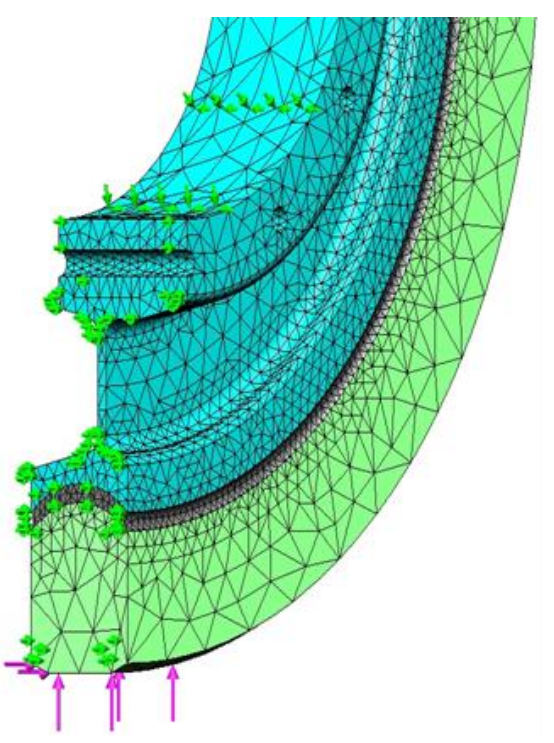

Figure 11 - Finite element mesh and boundary conditions of the supporting wheel (variant 3) with a diameter $780 \mathrm{~mm}$ 
The volume-stressed state of the supporting wheel Fig. 12 and the resulting displacement of the supporting wheel Fig. 13 were built on the results of modeling. According to Figure 12 it was found that under the action of radial and eccentric axle load the greatest stresses occur in the transition region of the rim inner ring to the thinner middle part of the wheel centre. Stresses reach $159 \mathrm{MPa}$, which is less than the allowable value (291.2 MPa). The stresses in the rubber pad increased to $2.5 \mathrm{MPa}$, which is much less than the tensile strength for this rubber brand (17 MPa).

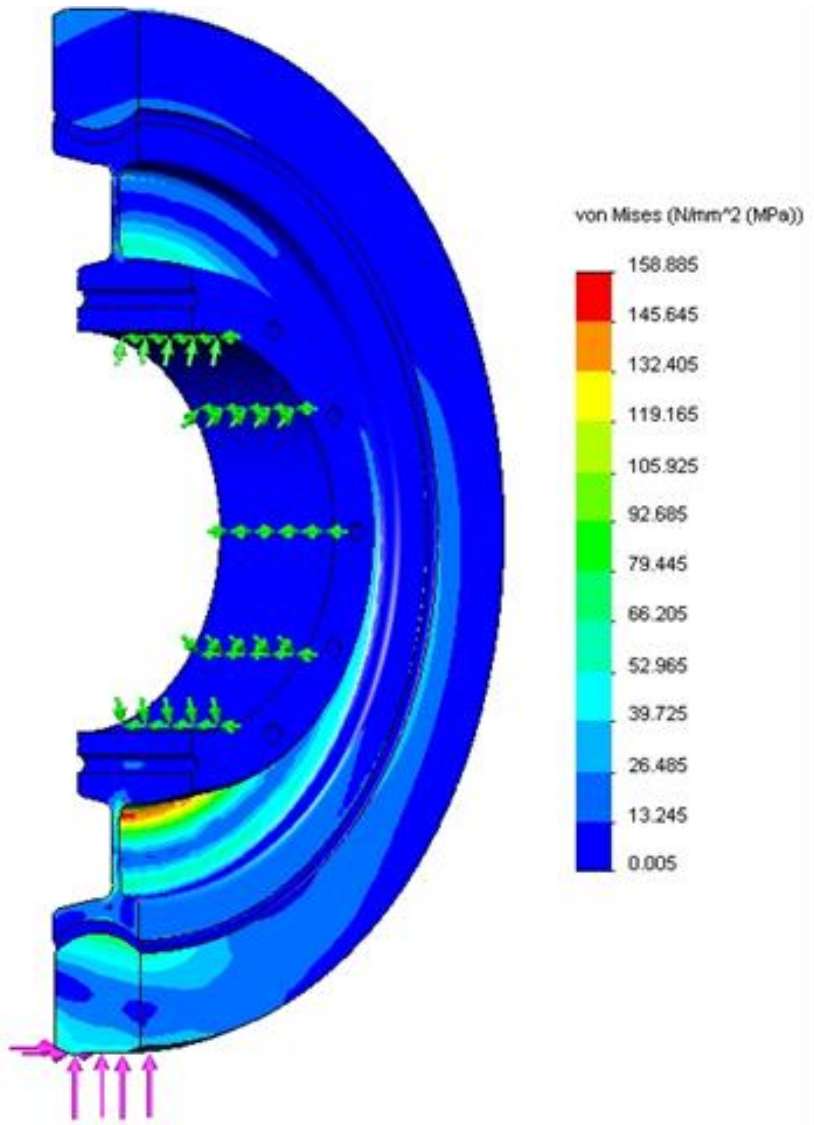

Figure 12 - Volume-stressed state of the supporting wheel (variant 3) with a diameter $780 \mathrm{~mm}$

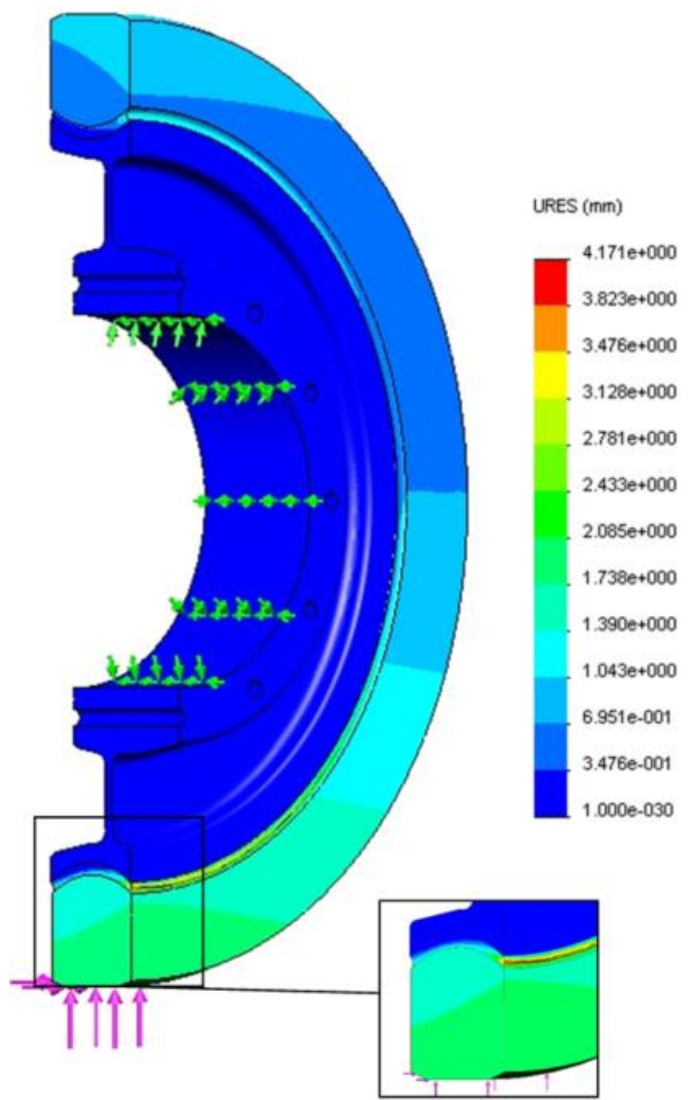

Figure 13 - Resulting displacements of the supporting wheel (variant 3) with a diameter $780 \mathrm{~mm}$

The supporting wheel stiffness became higher in the axial direction due to the reduction arm action of the shear force and the stresses in the supporting wheel elements are lower than in other variants [4]. The resulting displacement (Fig. 13) shows that the lower part of the flexible tire to which shear force is applied, is exposed to the greatest displacement. The axial displacement of the flexible tire bottom point has reduced compared to $4.1 \mathrm{~mm}$. In this case the gap between the resistant and supporting wheels enough to perform with the value $5 \mathrm{~mm}$.

The third variant with the reduced diameter of the flexible tire running surface $(780 \mathrm{~mm})$ has the lowest weight compared to the other variants in the case of a largest axial stiffness and smallest level of stresses in the wheel block elements. in Table 1

For a visual comparison of all the variants of a new wheelset design, their main strength characteristics are shown

\begin{tabular}{llllll}
\hline Variant & $\begin{array}{l}\text { Diameter } \\
\text { of the flexible } \\
\text { tire surface, } \\
\mathrm{mm}\end{array}$ & $\begin{array}{l}\text { Maximal stresses, } \\
\mathrm{MPa}\end{array}$ & $\begin{array}{l}\text { Maximal stresses } \\
\text { in the rubber pad, } \\
\mathrm{MPa}\end{array}$ & $\begin{array}{l}\text { Maximal axial } \\
\text { displacement of } \\
\text { the flexible tire, } \\
\mathrm{mm}\end{array}$ & Weight, kg \\
\hline 1 & 950 & 268 & 2.6 & 6 & 279 \\
2 & 950 & 253.5 & 2.5 & 4.4 & 270.8 \\
3 & 780 & 159 & 2.5 & 4.1 & 212.6 \\
\hline
\end{tabular}

Table 1. Technical specifications of the supporting wheel designs. 


\section{Conclusion}

1. Decision about the use of the standard wheelset "Py1-950" was made to improve the reliability in wheelset exploitation of the new design and reduce the cost of its manufacturing.

2. Wheel centre and flexible tire of the supporting wheel are made of the same steel as the standard wagon wheel centre.

3. The elastic pad between the wheel centre and flexible tire is made of a rubber material, which applies in a silent block of the journal box locking box of locomotive.

4. To improve the reliability of the flexible tire installation on the wheel centre, its mating surfaces them are made curved.

5. Flexible tire with the running surface diameter is $780 \mathrm{~mm}$ and corresponding to this diameter the other elements sizes of the wheel block are recommended to be used to weight reduction of the supporting wheel. In this case, the stress level at all of the power components of the wheel block is substantially lower in comparison with other variants of the wheelset designs.

\section{Reference}

[1] New wheelset design for rail transport, Bulletin of the Institute of Natural Monopoly Problems: Railways Equipment, Issn: 1998-9318, Moscow.

[2] I. Galiev, A. Shiler, A. Kildibekov, I. Kukushkin, High Speed Flexible Transport System, 25th DAAAM International Symposium On Intelligent Manufacturing And Automation, Vienna, Austria, 2015.

[3] A. Shiler, Analysis and Simulation of New Wheelset Construction, 25th DAAAM International Symposium On Intelligent Manufacturing And Automation, Vienna, Austria, 2015.

[4] C. Casanueva, A. Alonso, I. Eziolaza, G. Gimenez, Simple flexible wheelset model for low frequency instability simulations: Proceedings of the Institution of Mechanical Engineers, Part F: Journal of Rail and Rapid Transit 2012.

[5] B. Stefano, B. L. Gonzales, Flexible wheelset-track model for numerical estimation of stresses in railway axles, Anno Accademico 2009-2010. 